\title{
Forced oscillation technique for optimising PEEP in ventilated extremely preterm infants
}

\author{
To the Editor:
}

Ventilatory settings are critical in mechanically ventilated extremely preterm newborn infants due to the risk of ventilation-induced lung injury (VILI) and the subsequent development of bronchopulmonary dysplasia (BPD) [1]. Positive end-expiratory pressure (PEEP) settings usually rely on blood gases, oxygen requirement, lung auscultation, evaluation of chest radiograph and assessment of the pressure/volume curves provided by ventilators. Studies of optimal PEEP settings in the surfactant-treated preterm infant in need of mechanical ventilation are limited and evidence-based clinical guidelines are sparse [2, 3]. A bedside method identifying the PEEP value that comprises maximal lung volume recruitment and minimising tissue overdistension could improve real-time optimisation of PEEP and potentially minimise the risk of VILI and BPD $[4,5]$.

The respiratory input reactance measured by the forced-oscillation technique (FOT) has been shown to be feasible at the bedside in premature infants at different maturational and postnatal ages [6, 7]. In animal models, it has been shown to identify the lowest PEEP at which lung recruitment is most optimal during a decreasing PEEP trial $[8,9]$.

Our aim was to investigate the relationship between lung mechanics and PEEP during the first week of life using FOT, and to characterise the mechanical optimal PEEP (PEEP ${ }_{\mathrm{FOT}}$ ) settings in ventilated extremely preterm infants in comparison to the clinically set PEEP (PEEP $\left.{ }_{\text {CLIN }}\right)$.

Infants born at $<28$ weeks of gestational age were eligible if intubated and mechanically ventilated during the first day of life, and if no congenital abnormalities or other malformations relevant to lung function were present. During the study period, 22 patients met the inclusion criteria, but eight were excluded as they were extubated and remained so after day 1.14 extremely preterm infants (mean \pm SD gestational age $24.4 \pm 1.7$ weeks; birth weight $612 \pm 132 \mathrm{~g}$ ) were included after informed parental consent. All measurements were performed at the Neonatal Intensive Care Unit of the University Children's Hospital in Uppsala (Sweden) between June 2011 and May 2014. All infants received prenatal steroids and early surfactant. The median duration of mechanical ventilation was 38 days (range 12-239). One infant died before 36 weeks and seven (54\%) of the remaining 13 patients were diagnosed with severe BPD. The study was approved by the Regional Ethical Review Board (Dnr:99092).

Infants were intubated with uncuffed endotracheal tubes (ETT) $(2.0-2.5 \mathrm{~mm})$ and ventilated by assist/control ventilation (Stephanie Ventilator; Fritz Stephan GmbH, Gackenbach, Germany), enabling patients to receive support during spontaneous breaths. PEEP was chosen by the attending clinician, and the peak inspiratory pressures were set according to predefined carbon dioxide tension targets. During the intervention, ventilatory parameters except PEEP were kept constant, with fraction of inspired oxygen $\left(F_{\mathrm{IO}_{2}}\right)$ adjusted to maintain oxygen saturation according to institutional guidelines. The infants were studied on the 1st, 3rd and 7th day of life. Lung mechanics were measured using FOT with a 5, 10 and $15 \mathrm{~Hz}$ over-imposed sinusoidal pressure signal on the ventilation pressure waveform. PEEP was increased $2 \mathrm{cmH}_{2} \mathrm{O}$ above $\mathrm{PEEP}_{\mathrm{CLIN}}$, then decreased by four 5-minute steps of $1 \mathrm{cmH}_{2} \mathrm{O}$, and finally restored to PEEP $\mathrm{CLIN}_{\text {. Registration }}$ periods were held short so as to not interfere with the ongoing ventilatory assistance.

@ERSpublications

PEEP during ventilation of extremely preterm infants, evaluated by bedside forced oscillation technique in the first week of life, is lower than clinically set PEEP, suggesting that surfactant treated lungs can be easily overdistended even at low PEEP http://bit.ly/2FW1ZAQ

Cite this article as: Wallström L, Veneroni C, Zannin E, et al. Forced oscillation technique for optimising PEEP in ventilated extremely preterm infants. Eur Respir J 2020; 55: 1901650 [https://doi.org/10.1183/ 13993003.01650-2019]. 
Pressure was measured at the inlet of the ETT and the flow signal was obtained from the ventilator. All signals were sampled at $300 \mathrm{~Hz}$ by a custom-made digitiser and stored on a laptop for subsequent analysis. Reliable impedance-data were obtained from all frequencies without interference by ventilator frequency, but only data measured at $10 \mathrm{~Hz}$ is presented because 5 and $15 \mathrm{~Hz}$ did not provide more information on lung mechanics. All calculations were made after each registration and the attending clinicians were blinded to the results in all instances.

The respiratory system resistance $\left(R_{\mathrm{rs}}\right)$ and reactance $\left(X_{\mathrm{rs}}\right)$ were obtained by computing the transfer function between pressure and flow at the stimulus frequencies and correcting for the ETT contribution. $X_{\mathrm{rs}}$ accounts for the elastic and inertial properties of the system [10]. For each protocol step, 10 breaths were automatically selected for each frequency and their end-expiratory $R_{\mathrm{rs}}$ and $X_{\mathrm{rs}}$ values were averaged to have one data point for each PEEP level.

PEEP $_{\mathrm{FOT}}$ was defined as the pressure associated with the maximum value of $X_{\mathrm{rs}}$ during a decreasing PEEP trial $[6,8,11]$.

Two-way (days of life and PEEP) ANOVA for repeated measurements was used to test differences in the measured parameters. The Tukey test was used as a post hoc test after ANOVA. A p-value of $<0.05$ was considered statistically significant.

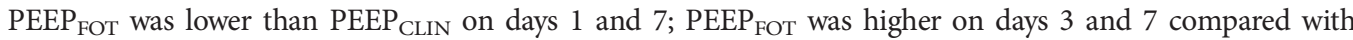
day 1, while $\mathrm{PEEP}_{\text {CLIN }}$ did not show statistical changes with time (figure 1 ). $X_{\mathrm{rs}}$ at $\mathrm{PEEP}$ FOT was higher than $X_{\mathrm{rs}}$ at $\mathrm{PEEP}_{\mathrm{CLIN}}$ (mean difference $(95 \% \mathrm{CI})$ of $X_{\mathrm{rs}}$ at $\mathrm{PEEP}_{\mathrm{FOT}}$ compared with $\mathrm{PEEP}_{\mathrm{CLIN}}$ was 4.7 (1.4-8.0) $\mathrm{cmH}_{2} \mathrm{O} \cdot \mathrm{s} \cdot \mathrm{L}^{-1}$ on day $1,2.5(0.4-4.6) \mathrm{cmH}_{2} \mathrm{O} \cdot \mathrm{s} \cdot \mathrm{L}^{-1}$ on day 3 and $5.2(0.04 ; 10.3) \mathrm{cmH}_{2} \mathrm{O} \cdot \mathrm{s} \cdot \mathrm{L}^{-1}$ on day 7$)$.

$X_{\mathrm{rs}}$ decreased significantly when PEEP was increased by $2 \mathrm{cmH}_{2} \mathrm{O}$ from $\mathrm{PEEP}_{\mathrm{CLIN}}$ (mean difference $(95 \% \mathrm{CI})$ 11.7 (8.3-15.2) $\mathrm{cmH}_{2} \mathrm{O} \cdot \mathrm{s} \cdot \mathrm{L}^{-1}$ on day $1 ; 10.0$ (5.7-14.4) $\mathrm{cmH}_{2} \mathrm{O} \cdot \mathrm{s} \cdot \mathrm{L}^{-1}$ on day 3 ; and $9.1(4.7-13.5) \mathrm{cmH}_{2} \mathrm{O} \cdot \mathrm{s} \cdot \mathrm{L}^{-1}$ on day 7) indicating that the lungs were easily overdistended. Oxygen need did not differ between the days or

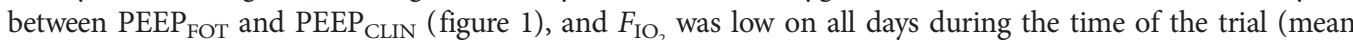
\pm SD $0.23 \pm 0.05,0.27 \pm 0.9$ and $0.26 \pm 0.06$, respectively, on days 1,3 and 7 ; p-value nonsignificant) resulting in nonsignificant changes in the oxygen saturation $/ F_{\mathrm{IO}_{2}}$ rates (figure 1). Clinically set peak inspiratory pressures were lower on the 1st day of life compared with the $3 \mathrm{rd}$ and 7 th day (mean \pm SD $15 \pm 2$ versus $18 \pm 4$ and 19 $\left.\pm 3 \mathrm{cmH}_{2} \mathrm{O} ; \mathrm{p}<0.05\right)$, but with no differences in tidal volumes $(4.4 \pm 0.9$ versus $4.7 \pm 0.8$ and $5.2 \pm 1.1 \mathrm{~mL})$ or respiratory rates $\left(59 \pm 8\right.$ versus $59 \pm 4$ and $58 \pm 9$ breaths $\cdot \mathrm{min}^{-1}$ ) between the study days.

In this cohort of extremely preterm infants, optimal PEEP as identified by FOT was lower than the clinically set PEEP on days 1 and 7. The lower optimal PEEP settings were not associated with changes in oxygenation or other clinical measurements compared with the clinically set PEEP. Our data suggests that clinically set PEEP levels might be too high, implying that the surfactant-treated lung is highly compliant and easily overdistended by PEEP during mechanical ventilation. Our data indicated overdistension of the lung despite the limited increase in PEEP during the trial $\left(+2 \mathrm{cmH}_{2} \mathrm{O}\right)$, and although only a small, but significant, mean difference was noted between PEEP $\mathrm{CLIN}_{\text {and }}$ PEEP $\mathrm{FOT}$ on days 1 and 7 , this represented a range of 0 to +2 and -1 to $+2 \mathrm{cmH}_{2} \mathrm{O}$ on these respective days. Also, on day 3, the individual differences between $\mathrm{PEEP}_{\mathrm{CLIN}}$ and $\mathrm{PEEP}_{\mathrm{FOT}}$ ranged from -1 to $+2 \mathrm{cmH}_{2} \mathrm{O}$. Thus, PEEP settings may be
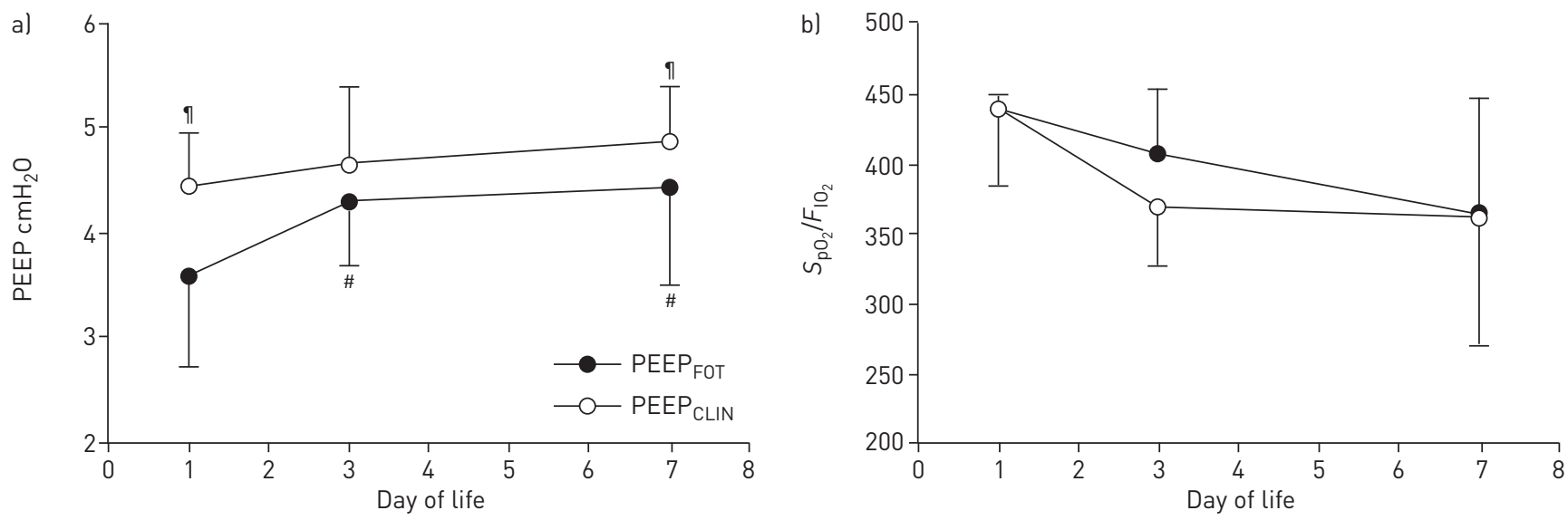

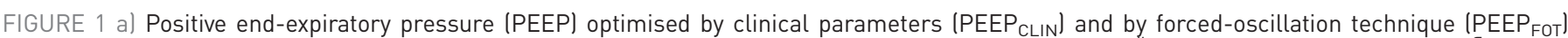
on the 1st, 3rd and 7th day of life. b) Oxygen saturation $\left(S_{\mathrm{pO}_{2}}\right)$ and fraction of inspired oxygen $\left(F_{\mathrm{IO}_{2}}\right)$ ratio. ${ }^{\#}:$ p $<0.05$ comparison to day 1 . ${ }^{\text {I: }}$ p $<<0.05$ comparison between PEEP CLIN $_{\text {and PEEP }}$ FOT. At day 3 PEEP $P_{\text {CIN }}$ and PEEP FOT did not reach statistical difference $(p=0.10)$. 
especially critical on the first day of life as we observed larger differences in $X_{\mathrm{rs}}$ during PEEP titration at the beginning than at the end of the first week of life.

The differences between PEEP derived from clinical data compared with PEEP derived from lung mechanical measurements by FOT, illustrates the limitation of the available bedside information for tailoring individualised PEEP and the need for more accurate assessment of lung mechanics during the first initial phase of life. The lower PEEP $\mathrm{FOT}$ was not associated with any changes in $F_{\mathrm{IO}_{2}}$, suggesting the uncertainty of using oxygen requirements to guide PEEP settings at bedside in extremely preterm newborn infants. Our data also suggests that in the absence of lung mechanical measurements, clinically set PEEP might be biased toward higher values, leading to potentially harmful overdistention of the immature lung.

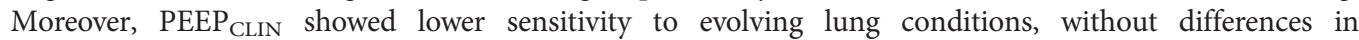
PEEP $_{\text {CLIN }}$ during the study period.

We have previously shown that bedside FOT is well tolerated in this population of extremely preterm infants during their 1st day of life [7]. This study adds information about evolving lung mechanics during the first postnatal week as measured by FOT, and we believe that $X_{\mathrm{rs}}$ changes with PEEP might provide useful information for real-time individualisation of PEEP repeatedly and on a daily basis.

Limitations of our study are the low number of patients included, the limited range of PEEP covered by the trial and the fixed inspiratory pressures (chosen to prevent changes in peak inspiratory pressures). In our present study, the impedance data were measured only at end-expiration, i.e. at PEEP, and it has been shown that optimal PEEP assessed by FOT minimises intratidal recruitment [9]. However, if the use of other ventilation approaches results in different applied tidal volumes, the degree of lung volume recruitment and, consequently, also $\mathrm{PEEP}_{\mathrm{FOT}}$, may change. Additionally, studies are needed to explore the effects of repeatedly adjusted PEEP settings according to FOT measurements on ventilation and oxygenation.

In summary, we have found that the optimal PEEP during mechanical ventilation of extremely preterm infants, as evaluated by FOT in the first week of life, is lower than the clinically set PEEP and that it changes over time. The lower PEEP $\mathrm{FOT}$ identified on day 1 compared with days 3 and 7 , together with the low $\mathrm{F}_{\mathrm{IO}_{2}}$ and peak inspiratory pressure, suggests that surfactant-treated lungs of extremely preterm infants can be easily overdistended even at low PEEP.

FOT might be a valuable bedside tool for assessment of lung mechanics for adjustments of PEEP over time and as part of a protective ventilation strategy to these vulnerable patients, especially during the first days of life. Further studies are needed to evaluate the possible long-term benefits of adjusting clinical PEEP by FOT, in this population of the most preterm infants.

Linda Wallström ${ }^{1,3}$, Chiara Veneroni ${ }^{2,3}$, Emanuela Zannin $\oplus^{2}$, Raffaele L. Dellacà $\oplus^{2}$ and Richard Sindelar ${ }^{1}$

${ }^{1}$ Dept of Women's and Children's Health, Uppsala University, Uppsala, Sweden. ${ }^{2}$ Dipartimento di Elettronica, Informazione e Bioingegneria, Politecnico di Milano, Milan, Italy. ${ }^{3}$ These authors contributed equally to the manuscript.

Correspondence: Linda Wallström, Dept of Women's and Children's Health, Uppsala University, Uppsala University Children’s Hospital, SE-751 85 Uppsala, Sweden. E-mail: linda.wallstrom@kbh.uu.se

Received: 21 Aug 2019 | Accepted after revision: 10 Jan 2020

Conflict of interest: L. Wallström has nothing to disclose. C. Veneroni reports that the Politecnico di Milano received research grants from Chiesi Farmaceutici SpA, Acutronic Medical Systems AG and Philips; and Politecnico di Milano owns a patent on the uses of the forced oscillation technique for monitoring lung volume recruitment; Politecnico di Milano also licensed patents on diagnostic application of the forced oscillation technique during mechanical ventilation to Acutronic Medical Systems AG and Philips. E. Zannin reports that the Politecnico di Milano received research grants from Chiesi Farmaceutici SpA, Acutronic Medical Systems AG and Philips; and Politecnico di Milano owns a patent on the uses of the forced oscillation technique for monitoring lung volume recruitment; Politecnico di Milano also licensed patents on diagnostic application of the forced oscillation technique during mechanical ventilation to Acutronic Medical Systems AG and Philips. R.L. Dellacà reports grants from Acutronic, outside the submitted work; in addition, R.L. Dellacà has a patent on the detection of EFL by FOT with royalties paid to Philips Respironics and Restech srl, a patent on monitoring lung volume recruitment by FOT with royalties paid to Vyaire, and a patent on early detection of exacerbations by home monitoring of FOT with royalties paid to Restech. R. Sindelar has nothing to disclose.

\section{References}

1 Kugelman A, Durand M. A comprehensive approach to the prevention of bronchopulmonary dysplasia. Pediatr Pulmonol 2011; 46: 1153-1165.

2 van Kaam AH, Rimensberger PC, Borensztajn D, et al. Ventilation practices in the neonatal intensive care unit: a cross-sectional study. J Pediatr 2010; 157: 767-771.

3 Bamat N, Fierro J, Wang Y, et al. Positive end-expiratory pressure for preterm infants requiring conventional mechanical ventilation for respiratory distress syndrome or bronchopulmonary dysplasia. Cochrane Database Syst Rev 2019; 2: CD004500.

4 Thome U, Töpfer A, Schaller P, et al. The effect of positive end expiratory pressure, and inspiratory time on functional residual capacity in mechanically ventilated preterm infants. Eur J Pediatr 1998; 157: 831-837. 
5 Mathe JC, Clement A, Chevalier JY, et al. Use of total inspiratory pressure-volume curves for determination of appropriate positive end-expiratory pressure in newborns with hyaline membrane disease. Intensive Care Med 1987; 12: 332-336.

6 Dellacà RL, Veneroni C, Vendettuoli V, et al. Relationship between respiratory impedance and positive end-expiratory pressure in mechanically ventilated neonates. Intensive Care Med 2013; 39: 511-519.

7 Veneroni C, Wallström L, Sindelar R, et al. Oscillatory respiratory mechanics on the first day of life improves prediction of respiratory outcomes in extremely preterm newborns. Pediatr Res 2019; 85: 312-317.

8 Dellacà RL, Zannin E, Kostic $\mathrm{P}$, et al. Optimisation of positive end-expiratory pressure by forced oscillation technique in a lavage model of acute lung injury. Intensive Care Med 2011; 37: 1021-1030.

9 Zannin E, Dellaca RL, Kostic P, et al. Optimizing positive end-expiratory pressure by oscillatory mechanics minimizes tidal recruitment and distension: an experimental study in a lavage model of lung injury. Crit Care 2012; 16: R217.

10 Dellacà RL, Kaczka DW. Oscillation mechanics of the respiratory system: applications to lung disease. Crit Rev Biomed Eng 2011; 39: 337-359.

11 Raffaeli G, Veneroni C, Ghirardello S, et al. Role of lung function monitoring by the forced oscillation technique for tailoring ventilation and weaning in neonatal ECMO: new insights from a case report. Front Pediatr 2018; 6: 332.

Copyright @eERS 2020 